\title{
Retroperitoneal Extraskeletal Osteosarcoma in a Pregnant Woman Incidentally Found on Cesarean Section
}

\author{
Shizue Fujihara ${ }^{1}$, Teruyuki Yamasaki ${ }^{1}$, Kontoku Shimokawa ${ }^{2}$, Han-Seung Yoon ${ }^{3}$ \\ ${ }^{1}$ Department of Obstetrics and Gynecology, lida Municipal Hospital, lida, Japan \\ ${ }^{2}$ Saint John of God Hospital Ballarat, Ballarat, Australia \\ ${ }^{3}$ Anatomic Pathology, lida Municipal Hospital, lida, Japan \\ Email: suzie.t0417@gmail.com
}

Received 18 November 2015; accepted 7 January 2016; published 11 January 2016

Copyright (C) 2016 by authors and Scientific Research Publishing Inc.

This work is licensed under the Creative Commons Attribution International License (CC BY). http://creativecommons.org/licenses/by/4.0/

(c) $\underset{\mathrm{EY}}{\mathrm{i}}$ Open Access

\begin{abstract}
Extraskeletal osteosarcoma (ESO) is a rare malignant mesenchymal neoplasm accounting for 1\% $2 \%$ of all soft tissue sarcomas that produce osteoid, bone, and/or chondroid material. Here we report the first case of a retroperitoneal ESO in a 35-year-old pregnant woman. A retroperitoneal ESO was incidentally found at cesarean section. Despite treatment, she rapidly died from wide spread dissemination. We focus on histological characteristics including immunohistochemical results and differential diagnosis of ESO.
\end{abstract}

Keywords

Extraskeletal Osteosarcoma, Pregnant Woman, MDM2, CDK4

\section{Introduction}

Extraskeletal osteosarcoma (ESO) is a rare malignant mesenchymal neoplasm accounting for $1 \%-2 \%$ of all soft tissue sarcomas that produce osteoid, bone, and/or chondroid material, and is located in soft tissues without attachment to bone or periosteum [1]. As far as we know, there is no report of ESO in a pregnant woman so far. Here we present a case report of a retroperitoneal ESO that was incidentally found on cesarean section in a pregnant woman.

\section{Case Report}

A 35-year-old woman, gravida 0, para 0 , presented to our hospital at 12 weeks' gestation. Her medical history

How to cite this paper: Fujihara, S., Yamasaki, T., Shimokawa, K. and Yoon, H.-S. (2016) Retroperitoneal Extraskeletal Osteosarcoma in a Pregnant Woman Incidentally Found on Cesarean Section. Open Journal of Pathology, 5, 14-18.

http://dx.doi.org/10.4236/ojpathology.2016.61003 
was unremarkable. She underwent a routine antenatal check which identified a non-functioning right kidney. However, because of her pregnancy, further imaging studies were not performed to limit radiation exposure to the unborn foetus. She was later admitted to our hospital at 37 weeks' gestation because of premature rupture of the membranes. Oxytocin was administered in order to augment the labor and delivery, however, finally a decision was made to proceed with a cesarean section because of arrest of labor.

Her preoperative blood tests were abnormal; alkaline phosphatase 2791 IU/l (normal range; 110 - 348), creatinine $2.67 \mathrm{mg} / \mathrm{dl}(0.5$ - 0.8), lactate dehydrogenase $376 \mathrm{IU} / \mathrm{l}(119$ - 221) and D-dimer $73.6 \mu \mathrm{g} / \mathrm{ml}(\leq 1)$ were high, and haemoglobin $9.2 \mathrm{~g} / \mathrm{dl}(11.1$ - 15.2) was low.

On entering the peritoneum, we found a large amount of bloody ascites. Immediately after the delivery of the baby, uncontrolled haemorrhage was encountered posterior to the uterus from what appeared to be a solid mass in the retroperitoneum. The mass was large, haemorrhagic and necrotic. It invaded the uterus, right ovary and appendix. The right ureter was also involved by the tumour, resulting in severe hydronephrosis. In order to stop the haemorrhage, an en-block resection of the tumour together with total hysterectomy, right salpingo-oophorectomy, appendectomy and partial right ureterectomy was performed. Estimated blood loss was 12,860 g, and the patient received a massive transfusion of 24 units of packed red blood cells, 20 units of fresh frozen plasma and 20 units of platelet concentrate. The postoperative course of the patient was uneventful and the patient was discharged on the ninth postoperative day.

On macroscopic findings, a large haemorrhagic mass measuring $15 \times 10 \times 8 \mathrm{~cm}$ in size (indicated by the dotted line in Figure 1(A)) was attached to the uterus (arrow) and right adnexa (arrowhead). The cut surface of the tumour (Figure 1(B)) showed a mass that was extensively necrotic and haemorrhagic with invasion to the outer myometrium. Pale white irregular areas within the tumour felt bone-like consistency. On microscopy, the tumour was a high-grade sarcoma with marked proliferation of atypical pleomorphic polygonal and short spindle cells. The tumour had geographical necrosis. There were numerous mitoses and a number of these appeared abnormal. Frequent areas showed formation of irregular hyalinised collagen matrix or immature osteoid that was often associated with osteoclast-type giant cells (Figure 2(A)). Of characteristic was that there were different stages of osteoid matrix formation throughout the tumour, and some of which showed calcification (Figure 2(B)). Other areas showed relatively mature lattice-like trabecular bone, consistent with those of a low-grade osteosarcoma (Figure 2(C)). The tumour therefore showed both high-grade and low-grade osteosarcoma. Immunohisto-chemistry showed that the tumour cells were positive for MDM2 (Figure 2(D)), CDK4 (Figure 2(E)), p16 and vimentin, focally positive for $\alpha$ SMA and negative for desmin. Ki-67 labelling index was over 50\%.
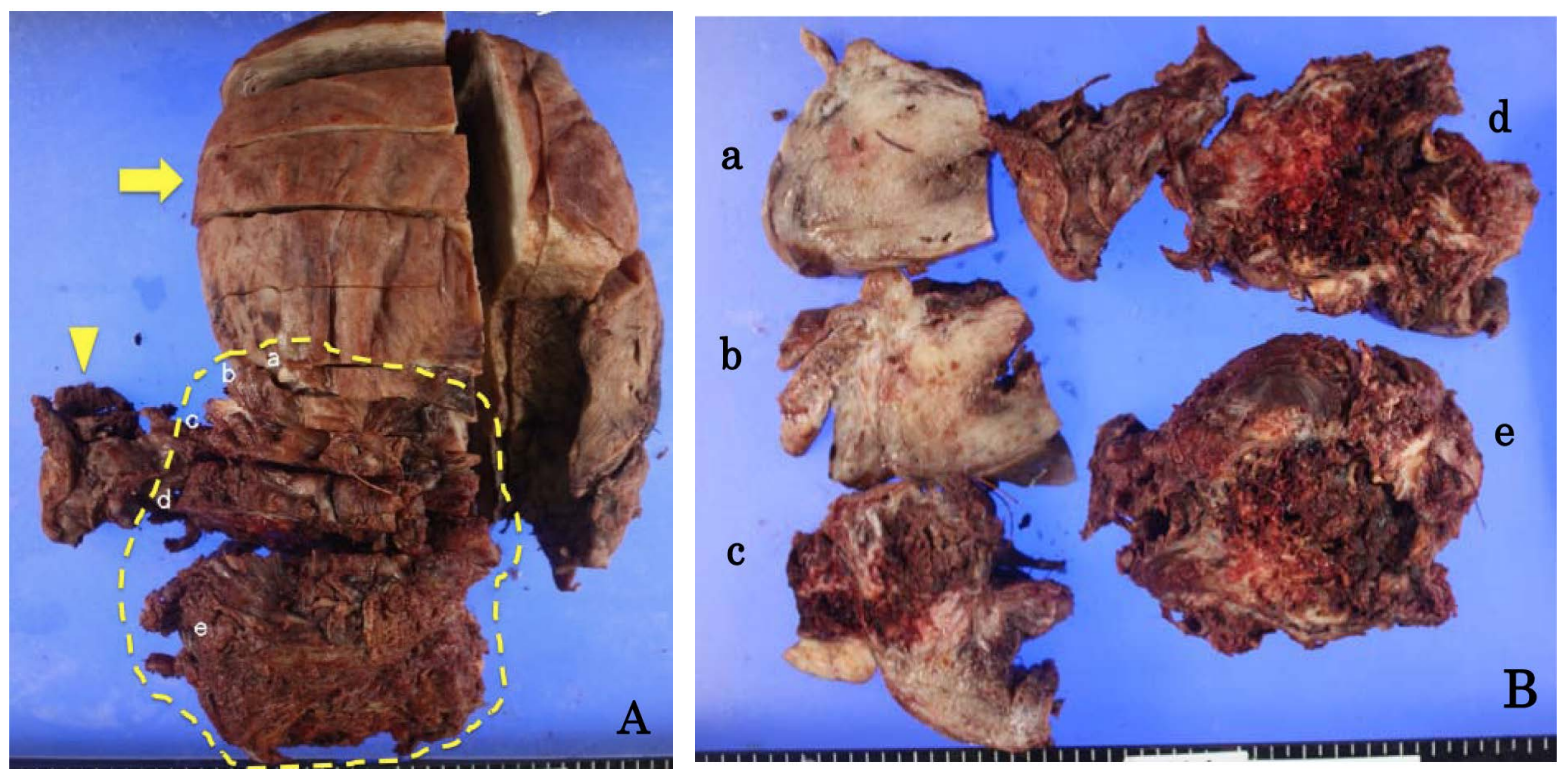

Figure 1. (A) The tumour (dotted frame region), uterus (arrow) and right adnexa (arrowhead). (B) The cut surface of the retroperitoneal mass and uterus showed a large, friable and necrotic tumour that invaded to the outer myometrium. Pale white irregular areas within the tumour represented issue with bony consistency. Lower-case characters (a-e) in (A) and (B) correspond to the same level of cut surfaces of the uterus and/or tumour. 



Figure 2. (A) Proliferation of atypical polygonal cells and short spindle cells forming irregular hyalinised collagen associated with osteoclast-type giant cells $(\mathrm{H} \& \mathrm{E}$, original magnification $\times 400)$; (B) Irregular osteoid matrix shows various stages of maturation and calcification $(\mathrm{H} \& \mathrm{E}$, original magnification $\times 200)$; (C) Prominent neoplastic new bone formation characterized by relatively mature trabecular bone $(\mathrm{H} \& \mathrm{E}$, original magnification $\times 200)$; (D) Occasional tumour cells show strong nuclear positivity for MDM2 while many cells show mild to moderate positivity (MDM2, original magnification $\times 200$ ); (E) Many cells show moderate positivity for CDK4 (CDK4, original magnification $\times 200)$.

The tumour invaded to the outer myometrium, periappendiceal tissue and right ureter resulting in right hydronephrosis. The endometrium was unremarkable except for changes associated with gestation. The tumour showed no connection with pre-existing bony structures. Based on these findings a final diagnosis of ESO originating from 


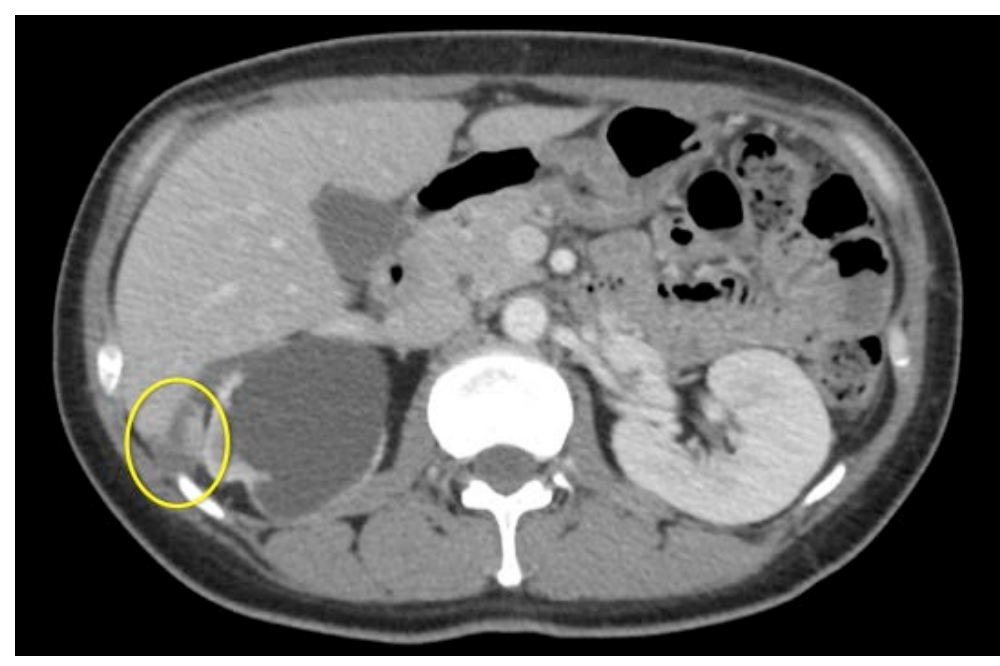

Figure 3. CT scan performed on post-operative day 17 revealed peritoneal dissemination in Morrison's pouch, which measured $23 \times 14 \mathrm{~mm}$ in size (framed region).

the retroperitoneum was made.

CT scan performed on post-operative day 17 revealed peritoneal dissemination in Morrison's pouch, which measured $23 \times 14 \mathrm{~mm}$ in size (Figure 3). Once again, there were no lesions seen in the bone.

On post-operative day 36, she was re-admitted because of abdominal fullness due to the accumulation of ascitic fluid. Three liters of bloody ascites was removed. CT scan showed that the mass in the Morrison's pouch had enlarged to $97 \times 75 \mathrm{~mm}$ in size. Several other masses were also seen in the peritoneum and greater omentum. The patient received cisplatin, doxorubicin and pazopanib with no benefit. She died 6 months after the surgery.

\section{Discussion}

Here we have described a rare retroperitoneal ESO in a young pregnant woman incidentally found at cesarean section, who rapidly died from wide spread dissemination. Although skeletal osteosarcoma has been reported in pregnant women [2], as far as we know, this is the first report of an ESO in a pregnant woman.

In addition, of interest in this patient, was that the tumour cells were strongly positive for MDM2, CDK4 and p16. A recent study reported that $6 \%-8 \%$ of primary and recurrent/metastatic high-grade skeletal osteosarcoma immunohistochemically expressed MDM2 and CDK4 [3]. These authors found that most tumours with the coexpression of the immunohistochemistry markers contained foci of coexistent low-grade osteosarcoma, whereas none of high-grade osteosarcoma lacking the coexpression contained foci of low-grade osteosarcoma. Thus the authors suggested that MDM2 and CDK4 coexpression was specific to a subset of high-grade osteosarcoma that had progressed from a low-grade precursor. Our present ESO case seems to support these findings with respect to immunohistochemistry and differentiation features.

It has recently been reported that dedifferentiated liposarcoma characteristically harbours MDM2 and CDK4 coamplification and coexpression [4]. Therefore it was mandatory to distinguish between ESO and dedifferentiated liposarcoma since the 2 tumours showed similar undifferentiated spindle cell characteristics. The present case showed extensive new bone formation by tumour cells ranging from irregular small hyalinised collagen or osteoid through to relatively mature larger osteoid with early calcification to mature lattice-like trabecular bone structure. Furthermore, there was no obvious lipomatous component within or adjacent to the tumour. Based on these findings, we favoured a diagnosis of ESO rather than dedifferentiated liposarcoma. Other possibilities such as uterine or tubal sarcomas are unlikely because the tumour was centered in the retroperitoneum.

ESO occurs in older adults compared with skeletal osteosarcoma. The median age for ESO is reported between 44 - 55 years old, and mostly instances occur in adults over the age of 30 years old [1] [5] [6]. The most common location for primary ESO is the soft tissues of the thigh (27.3\%), followed by the upper extremity (20.5\%) and the retroperitoneum (17\%) [1]. A history of prior trauma or radiation therapy is reported in $12.5 \%$ or $5 \%$ of patients, respectively [1]. 
ESO is a very aggressive tumour with poor prognosis. The reported rates were $19 \%$ - $69 \%$ for local recurrence and $37 \%-80 \%$ for distant metastases [6]-[8]. Overall survival rates at 5 years are poor ranging from $25 \%$ to $77 \%$ [5] [8]. Previous studies have shown large tumour size $(<5$ versus $\geq 5 \mathrm{~cm})$, positive margins and deep tumours are to be significant prognostic factors [6] [7] [9]. Choi et al. concluded that neither radiation nor chemotherapeutic treatment were associated with a lower incidence of death due to disease or a longer event-free survival [7].

In our case, tumour cells were strongly positive for MDM2 and CDK4, which supported the recent findings that MDM2 and CDK4 coexpression was specific to a subset of high-grade osteosarcoma that had progressed from a low-grade precursor.

\section{Acknowledgements}

This case report was approved by the patient's family’s consent.

\section{References}

[1] Chung, E.B. and Enzinger, F.M. (1987) Extraskeletal Osteosarcoma. Cancer, 60, 1132-1142. http://dx.doi.org/10.1002/1097-0142(19870901)60:5<1132::AID-CNCR2820600536>3.0.CO;2-L

[2] Corrêa, R.R., Espindula, A.P., Machado, J.R., Paschoini, M.C., Pacheco Olegário, J.G., Rocha, L.P. and dos Reis, M.A. (2012) Osteosarcoma in a Pregnant Woman: Case Report. Archives of Gynecology and Obstetrics, 286, 1601-1602. http://dx.doi.org/10.1007/s00404-012-2483-3

[3] Yoshida, A., Ushiku, T., Motoi, T., Beppu, Y., Fukayama, M., Tsuda, H. and Shibata, T. (2012) MDM2 and CDK4 Immunohistochemical Coexpression in High-Grade Osteosarcoma: Correlation with a Dedifferentiated Subtype. American Journal of Surgical Pathology, 36, 423-431. http://dx.doi.org/10.1097/PAS.0b013e31824230d0

[4] Thway, K., Flora, R., Shah, C., Olmos, D. and Fisher, C. (2012) Diagnostic Utility of p16, CDK4, and MDM2 as an Immunohistochemical Panel in Distinguishing Well-Differentiated and Dedifferentiated Liposarcomas from Other Adipocytictumours. American Journal of Surgical Pathology, 36, 462-469.

http://dx.doi.org/10.1097/PAS.0b013e3182417330

[5] Goldstein-Jackson, S.Y., Gosheger, G., Delling, G., Berdel, W.E., Exner, G.U., Jundt, G., Machatschek, J.N., Zoubek, A., Jürgens, H. and Bielack, S.S. (2005) Extraskeletal Osteosarcoma Has a Favourable Prognosis When Treated Like Conventional Osteosarcoma. Journal of Cancer Research and Clinical Oncology, 131, 520-526. http://dx.doi.org/10.1007/s00432-005-0687-7

[6] Ahmad, S.A., Patel, S.R., Ballo, M.T., Baker, T.P., Yasko, A.W., Wang, X., Feig, B.W., Hunt, K.K., Lin, P.P., Weber, K.L., Chen, L.L., Zagars, G.K., Pollock, R.E., Benjamin, R.S. and Pisters, P.W.T. (2002) Extraosseous Osteosarcoma: Response to Treatment and Long-Term Outcome. Journal of Clinical Oncology, 20, 521-527. http://dx.doi.org/10.1200/JCO.20.2.521

[7] Choi, L.E., Healey, J.H., Kuk, D. and Brennan, M.F. (2014) Analysis of Outcomes in Extraskeletal Osteosarcoma: A Review of Fifty-Three Cases. The Journal of Bone \& Joint Surgery, 96, e2. http://dx.doi.org/10.2106/JBJS.M.00339

[8] Sordillo, P.P., Hajdu, S.I., Magill, G.B. and Golbey, R.B. (1983) Extraosseous Osteogenic Sarcoma. A Review of 48 Patients. Cancer, 51, 727-734. http://dx.doi.org/10.1002/1097-0142(19830215)51:4<727::AID-CNCR2820510429>3.0.CO;2-I

[9] Bane, B.L., Evans, H.L., Ro, J.Y., Carrasco, C.H., Grignon, D.J., Benjamin, R.S. and Ayala, A.G. (1990) Extraskeletal Osteosarcoma: A Clinicopathologic Review of 26 Cases. Cancer, 66, 2762-2770. http://dx.doi.org/10.1002/1097-0142(19900615)65:12<2762::AID-CNCR2820651226>3.0.CO;2-K 